\title{
Tritium and cancer risk: incorporating informative priors from experimental research into epidemiology
}

\author{
David B. Richardson ${ }^{1}$ \\ ${ }^{1}$ School of Public Healthn University of North Carolina at Chapel Hill, Chapel Hill, NC, USA
}

The health effects of exposure to tritium, a radioactive isotope of hydrogen, are of interest for a number of reasons: exposure is widespread, and includes planned and unplanned releases from nuclear sites; tritium is very mobile; and there remains uncertainty about its biological effects. There have been a number of authoritative reviews of tritium and the experimental evidence regarding its biological effects. These have tended to reach similar conclusions, suggesting relative biological effectiveness values in the range of 1.1-5.0 when compared with chronically-delivered gamma rays; a number of reports have concluded that that tritium beta particles should be expected to be at least as biologically effective as gamma rays. Evidence of tritium effects on human health derived through epidemiological studies, while arguably the most direct way to address questions about tritium health effects, unfortunately, is very limited. This has led some authors to contend that this limited epidemiological evidence means it is necessary to derive estimates of tritium risk indirectly from experimental studies. An alternative viewpoint, however, is to acknowledge the limits of the available evidence (human and experimental) and therefore integrate these lines of evidence, proposing that neither on its own is sufficient to address the question, but combined we may usefully address the question. A major limitation of the epidemiological data is that sparse data make it difficult to obtain reliable estimates of association. In settings of sparse data, Bayesian inference with informative priors can be used to improve estimation of associations. What is that prior information, and how should we incorporate it? As reviewed in a recent US NCRP report, a variety of lines of evidence) pertain to the biological effectiveness of tritium in experimental settings. These tend to suggest that low-energy beta emitters will have greater biological effectiveness per unit absorbed dose than standard reference radiations under most circumstances. We have proposed to incorporate such evidence as a prior, implemented with what is known as an ordered constrained prior. Under this prior, we posit that the effect of tritium per unit absorbed dose is at least as effective as external photons (primarily gamma radiation) per unit absorbed dose. We have illustrated this in a study of radiation dose and leukemia mortality among 18,883 workers at the Savannah River Site employed from 1951-1999 and followed through 2002. The approach yields a posterior estimate of the excess relative rate per $100 \mathrm{mGy}$ for external photon exposure and an estimate of the excess relative rate per $100 \mathrm{mGy}$ for tritium intakes. The ratio of these estimates suggests a relative effectiveness of approximately 4, a value that falls within the range of estimates obtained from experimental studies (e.g., $1.1-5$ ), and suggests a potentially useful direction for further integration of experimental and epidemiological lines of evidence regarding tritium health effects. 